\title{
Model of Interacting Spin One Bosons
}

\author{
M. Corgini ${ }^{1, *}$, D. P. Sankovich ${ }^{2}$ \\ ${ }^{1}$ Departamento de Matemáticas, Universidad de La Serena, Cisternas 1200, La Serena, Chile \\ ${ }^{2}$ Steklov Mathematical Institute, Gubkin str. 8, 119991, Moscow, Russia \\ *Corresponding Author: mcorgini@userena.cl
}

Copyright (C)2014 Horizon Research Publishing All rights reserved.

\begin{abstract}
Within Bogolyubov approximating Hamiltonian approach the limit pressure is found in a system of atoms with internal three-level structure (spin-1 system). Moreover, the emergence of a Bose-Einstein condensation is proved for some special case of coupling constants.
\end{abstract}

Keywords Bose-Einstein condensation, Approximating Hamiltonian, Pressure, Spectrum gap

\section{Introduction}

\subsection{Second quantized Hamiltonian for spin-1 Bose systems}

Atoms in a spin-1 state are characterized by a vectorial field operator with its components subject to a hyperfine spin manifold [1]. The components of the field

$$
\Psi(\mathbf{r})=\left(\Psi_{1}(\mathbf{r}), \Psi_{2}(\mathbf{r}), \Psi_{3}(\mathbf{r})\right)^{\mathrm{T}}, \quad \mathbf{r} \in \mathbb{R}^{3}
$$

where the superscript $\mathrm{T}$ denotes the transpose, satisfy the usual Bose commutation rules $\left[\Psi_{i}(\mathbf{r}), \Psi_{j}\left(\mathbf{r}^{\prime}\right)\right]=$ $\Psi_{i}(\mathbf{r}) \Psi_{j}\left(\mathbf{r}^{\prime}\right)-\Psi_{j}\left(\mathbf{r}^{\prime}\right) \Psi_{i}(\mathbf{r})=\delta_{i, j} \delta\left(\mathbf{r}-\mathbf{r}^{\prime}\right)$ where $\delta_{i, j}$ and $\delta\left(\mathbf{r}-\mathbf{r}^{\prime}\right)$ are the Kronecker delta and the Dirac distribution, respectively.

The interaction Hamiltonian representing the atom system in terms of three spin components can be expressed explicitly as $[2,3,4]$

$$
\begin{gathered}
H_{\mathrm{int}}=\frac{1}{2} \int d^{3} r\left[\left(c_{0}+c_{2}\right) \Psi_{1}^{+} \Psi_{1}^{+} \Psi_{1} \Psi_{1}+c_{0} \Psi_{0}^{+} \Psi_{0}^{+} \Psi_{0} \Psi_{0}\right. \\
+\left(c_{0}+c_{2}\right) \Psi_{-1}^{+} \Psi_{-1}^{+} \Psi_{-1} \Psi_{-1}+2\left(c_{0}+c_{2}\right) \Psi_{1}^{+} \Psi_{0}^{+} \Psi_{0} \Psi_{1} \\
+2\left(c_{0}-c_{2}\right) \Psi_{1}^{+} \Psi_{-1}^{+} \Psi_{-1} \Psi_{1}+2\left(c_{0}+c_{2}\right) \Psi_{0}^{+} \Psi_{-1}^{+} \Psi_{-1} \Psi_{0} \\
\left.\quad+2 c_{2} \Psi_{-1}^{+} \Psi_{1}^{+} \Psi_{0} \Psi_{0}+2 c_{2} \Psi_{0}^{+} \Psi_{0}^{+} \Psi_{-1} \Psi_{1}\right],
\end{gathered}
$$

where $c_{0}, c_{1}, c_{2}$ are real parameters associated to twobody interaction strengths. In this interaction operator, the first three terms represent the intrastate collisions, or the self-scattering, the following three terms represent the interstate collisions or the cross-scattering, and the last two terms stand for the spin mixing.

In the next sections we shall study a system of bosonic atoms with internal degrees of freedom whose energy operator contains cross-scattering and self-scattering terms and in absence of spin mixing. The case of atoms in two different hyperfine states (spin-1/2), confined in a hypercube was analyzed in three early works $[5,6,7]$ in the framework of the approximating Hamiltonian methods and large deviations methods.

\subsection{Spectrum gap for the free Laplacian}

The behavior of Bose systems at the boundary of its confinement region of volume $V$ (for example, a cubic box of side $\ell$ ) is a fundamental problem in the study of many particle systems. In the case of a cubic box the particles can be repelled, attracted or reflected by the boundaries.

The energy of a gas of non-interacting Bose particles is described by the free Laplacian operator $\triangle_{\ell}$ under suitable boundary conditions and its eigenvalues $E$, representing the energy levels of the system, are obtained from the equation $-\triangle_{\ell} \phi / 2=E \phi$, where $\phi$ are the free Laplacian eigenfunctions.

We have to point out that different kinds of boundary conditions have been extensively studied for the free Bose gas for example in refs. [8, 9, 10].

The attractive boundary conditions are given by the relation

$$
\frac{\partial \phi}{\partial n}+\chi \phi=0
$$

where $\partial \phi / \partial n$ is the outward normal derivative on the boundary, being $\chi<0$ the attraction strength parameter. In this case, the spectrum of the Laplacian presents a gap. In other words, the lowest energy (which is $-\nu \chi^{2}$ in the thermodynamic limit, where $\nu$ is the dimension of the system) is a non-vanishing (negative) isolated point of the spectrum. This fact yields to condensation in any dimensions differently from the cases of Neumann, Dirichlet and periodic boundary conditions for which Bose-Einstein condensation (BEC) only holds for $\nu \geq 3$. In fact, when $\chi<0$ we are in presence of a not homogeneous condensate mostly concentrated near the boundaries, i.e. many of its particles bind to the container walls. Thus the attractive wall condensation is a surface effect rather than the usual bulk phenomenon obtained under other boundary conditions. In realistic scenarios such as a not homogeneously distributed in space trapped gas the excitation spectrum is separated from the ground state [11]. Moreover, models with attractive boundary conditions are extremely useful in the study of 
wetting phase transitions in a superconductor [12] and for proving the existence of super-surface films in liquid helium II [13]. On the other hand, in the homogeneous cases any spectral gap vanishes in the thermodynamic limit.

Many authors have considered the idea of introducing a spectral gap between the ground state and the excited states in the case of some Bose gases, including the ideal one, for studying condensation in different scenarios. In this sense, in refs. $[14,15,16]$ a homogeneous Bose gas with periodic boundary conditions and a two-body interaction enclosed in a region of volume $V$ has been exhaustively studied. In this works the term $-\triangle \hat{a}_{\mathbf{0}}^{\dagger} \hat{a}_{\mathbf{0}}$, where $\triangle>0$ and $\hat{a}_{\mathbf{0}}^{\dagger}, \hat{a}_{\mathbf{0}}$ are the creation and annihilation operators of the zero mode, was added to the corresponding energy operator, shifting in this way the zero energy level of the kinetic energy operator to a negative value and generating a gap in the spectrum. Thus, it was proved the emergence of BEC at low temperatures for a class of superstable interacting systems at low enough temperatures, large enough particle densities and spectral gap.

For example, for the superstable mean field type model whose energy operator is given by

$$
\hat{H}_{b}^{\triangle}=\hat{H}^{0, \triangle}+\frac{b}{2 V} \hat{N}^{2},
$$

where $b>0, V$ is the volume enclosing the particle system, $\hat{H}^{0, \triangle}$ and $\hat{N}$ are the free energy operator including a spectral gap and the total number operator, respectively, in ref. [15] it is derived the following expression for the limit pressure $p_{b}^{\triangle}(\beta, \mu)$ of the model:

$p_{b}^{\triangle}(\beta, \mu)= \begin{cases}p_{b}^{\triangle=0}(\beta, \mu), & \mu \leq-\triangle+b \rho^{\mathrm{p}}(\beta,-\triangle), \\ \frac{(\mu+\triangle)^{2}}{2 b}+p^{\mathrm{p}}(\beta,-\triangle), & \mu>-\triangle+b \rho^{\mathrm{p}}(\beta,-\triangle),\end{cases}$ where $p^{\mathrm{P}}(\beta, \mu)$ and $\rho^{\mathrm{P}}(\beta, \mu)\left(\beta\right.$-inverse temperature, $\mu^{-}$ chemical potential) are the pressure and the total density of the ideal Bose gas, respectively. In this case the amount of condensate is

$\rho_{0, b}^{\triangle}(\beta, \mu)= \begin{cases}0, & \mu \leq-\triangle+b \rho^{\mathrm{p}}(\beta,-\triangle), \\ \frac{\mu+\triangle}{b}-\rho^{\mathrm{p}}(\beta,-\triangle), & \mu>-\triangle+b p^{\mathrm{p}}(\beta,-\triangle) .\end{cases}$

The limit $\triangle \downarrow 0$ leads to

$$
\rho_{0, b}^{\triangle=0}(\beta, \mu)= \begin{cases}0, & \mu \leq b \rho_{\mathrm{c}}^{\mathrm{p}}(\beta), \\ \frac{\mu}{b}-p_{\mathrm{c}}^{\mathrm{p}}(\beta), & \mu>b p_{\mathrm{c}}^{\mathrm{p}}(\beta),\end{cases}
$$

where $p_{\mathrm{c}}^{\mathrm{p}}(\beta)$ is the critical density of BEC for the ideal gas. Clearly, the independent on temperature terms in the expressions for pressure and amount of condensate are consequence of the presence of the gap in the energy spectrum of the free energy operator. The amount of condensate still depends on temperature. Noting that $\hat{N}^{2}=\hat{N}^{\prime 2}+2 \hat{N}^{\prime} \hat{n}_{\mathbf{0}}+\hat{n}_{\mathbf{0}}^{2}$, where $\hat{n}_{\mathbf{0}}, \hat{N}^{\prime}$ are the operator number of particles associated to the low energy level and the total number operator with exclusion of $\hat{n}_{\mathbf{0}}$, respectively, neglecting collisions between particles in the condensate and the rest of the system particles and assuming that the low energy level is equal to zero, in the case of periodic boundary conditions we get a superstable model with energy operator

$$
\hat{H}_{b}^{\triangle}=\hat{H}^{0, \triangle}+\frac{b}{2 V} \hat{N}^{\prime 2} .
$$

In this case a macroscopic occupation of the ground state does not occur. However, under attractive boundary conditions or by introducing a spectrum gap in the excitation spectrum of the free energy operator the above situation changes dramatically since an independent on temperature macroscopic occupation of the ground state takes place. In this sense, we are in presence of a different kind of condensation - non-conventional BEC (see, for example, works $[5,6,7,17,18,19,20]$ for further details).

\subsection{Paper organization}

In earlier papers we take different approaches such as large deviations [7] and approximating Hamiltonian $[5,6]$ strategies for obtaining the limit pressures in the case of Bose-atom systems with internal two level (spin-1/2) an one level (spin-0) structures. This enabled us to prove interesting results related to nonconventional or independent on temperature BEC in the case of Bose systems whose ground states are associated to negative values of energies. In this paper we extend these results to the systems with three internal levels and negative minimal energies. In section 2 we introduce the model of the interacting Bose gas that will be studied. It corresponds to a homogeneous system with a gap at the excitations spectrum of its free part, analogous to such one discussed in refs. [14, 15, 16]. An approximating Hamiltonian is constructed and the coincidence of the limit pressures, in the sense of Wentzel, of the original model and the approximate one is demonstrated. In this case, the emergence of non-conventional BEC is proven when the coupling constants associated to intrastate collisions take the same positive value. Finally, section 3 is devoted to some discussion of the results.

\section{Non-conventional BEC spin-1 Bose model}

\subsection{General setting}

The one-particle free Hamiltonian corresponds to the operator $T^{\ell}=-\triangle / 2$ defined on a dense subset of the Hilbert space $\mathcal{H}^{\ell}=L^{2}\left(\Lambda_{\ell}\right)$, where $\Lambda_{\ell}=[-\ell / 2, \ell / 2]^{d} \subset$ $\mathbb{R}^{d}$ is a cubic box of boundary $\partial \Lambda_{\ell}$ and volume $V_{\ell}=\ell^{d}$. We assume periodic boundary conditions under which $T^{\ell}$ is self-adjoint.

We consider a system of Bose atoms with an internal three level structure. In this case any one-particle wave function has the form $\phi \otimes s$ where, $\phi \in L^{2}\left(\Lambda_{\ell}\right)$ and $s \in \mathcal{S}$, where

$$
\mathcal{S}=\left\{\left(\begin{array}{l}
1 \\
0 \\
0
\end{array}\right),\left(\begin{array}{l}
0 \\
1 \\
0
\end{array}\right),\left(\begin{array}{l}
0 \\
0 \\
1
\end{array}\right)\right\}
$$

represents the internal state. Therefore the vector space associated to this system is in fact, $\mathcal{H}_{s}^{\ell}=L^{2}\left(\Lambda_{\ell}\right) \otimes \mathcal{S}$.

$$
\begin{aligned}
\hat{H}_{\ell}(\mu) & =\hat{H}_{\ell}^{0}(\mu)+\frac{a}{V_{l}} \sum_{\sigma, \mathbf{p}}\left(\hat{a}_{\mathbf{p}, \sigma}^{\dagger}\right)^{2} \hat{a}_{\mathbf{p}, . \sigma}^{2} \\
& +\frac{1}{V_{\ell}} \sum_{\sigma_{1}<\sigma_{2}} \gamma_{\sigma_{1}, \sigma_{2}} \hat{n}_{\mathbf{0}, \sigma_{1}} \hat{n}_{\mathbf{0}, \sigma_{2}}+V_{\ell} g\left(\frac{\hat{N}^{\prime}}{V_{\ell}}\right)(1)
\end{aligned}
$$


where $\sigma=1,2,3, \gamma_{\sigma_{1}, \sigma_{3}}>0 \quad\left(\sigma_{1}<\sigma_{2}\right), a>0$. $g: \mathbb{R}^{+} \cup\{0\} \rightarrow \mathbb{R}^{+} \cup\{0\}$ is a differentiable increasing function such that $g(0)=0, g(x) \geq x^{2}$. The Davies type interaction operator $V_{\ell} g\left(\hat{N}^{\prime} / V_{\ell}\right)$ [21] ensures the superstability of this model.

The sum in eq. (1) runs over the set $\Lambda_{\ell}^{*}=\{\mathbf{p}=$ $\left(p_{1}, \ldots, p_{d}\right) \in \mathbb{R}^{d}: p_{\alpha}=2 \pi n_{\alpha} / \ell, n_{\alpha} \in \mathbb{Z}, \alpha=$ $1,2, \ldots, d\} . \quad \hat{a}_{\mathbf{p}, \sigma}^{\dagger}, \hat{a}_{\mathbf{p}, \sigma}$ are the Bose operators of creation and annihilation of particles defined on the Bose Fock space $\mathcal{F}_{\mathrm{B}}$. The usual commutation rules are satisfied: $\left[\hat{a}_{\mathbf{q}, \sigma_{1}}, \hat{a}_{\mathbf{p}, \sigma_{2}}^{\dagger}\right]=\hat{a}_{\mathbf{q}, \sigma_{1}} \hat{a}_{\mathbf{p}, \sigma_{2}}^{\dagger}-\hat{a}_{\mathbf{p}, \sigma_{2}}^{\dagger} \hat{a}_{\mathbf{q}, \sigma_{1}}=\delta_{\mathbf{p}, \mathbf{q}} \delta_{\sigma_{1}, \sigma_{2}}$. $\hat{n}_{\mathbf{p}, \sigma}=\hat{a}_{\mathbf{p}, \sigma}^{\dagger} \hat{a}_{\mathbf{p}, \sigma}$ is the number operator associated to mode $\mathbf{p}$ and internal level $\sigma$. In this case $\hat{H}_{\ell}^{0}(\mu)=$ $\sum_{\mathbf{p} \in \Lambda_{\ell}^{*}, \sigma}\left(\lambda_{\ell}(\mathbf{p}, \sigma)-\mu\right) \hat{n}_{\mathbf{p}, \sigma}, \quad a>0 . \hat{N}=\sum_{\mathbf{p} \in \Lambda_{\ell}^{*}, \sigma} \hat{a}_{\mathbf{p}, \sigma}^{\dagger} \hat{a}_{\mathbf{p}, \sigma}$ is the total number operator, $\hat{N}^{\prime}=\sum_{\mathbf{p} \in \Lambda_{\ell}^{*} \backslash\{\mathbf{0}\}, \sigma} \hat{a}_{\mathbf{p}, \sigma}^{\dagger} \hat{a}_{\mathbf{p}, \sigma}$ is the total number operator with exclusion of $\hat{n}_{\mathbf{0}, 1}, \hat{n}_{\mathbf{0}, 2}$, $\hat{n}_{\mathbf{0}, 3}$ and $\lambda_{\ell}(\mathbf{0}, 1)=-\lambda_{1}, \lambda_{\ell}(\mathbf{0}, 2)=0, \lambda_{\ell}(\mathbf{0}, 3)=-\lambda_{3}$ for $\mathbf{p}=\mathbf{0}, \lambda_{\ell}(\mathbf{p}, 1)=\mathbf{p}^{2} / 2$ for $\mathbf{p} \neq \mathbf{0}$, where $\lambda_{1}, \lambda_{3}>0$.

Note that the boson Fock space $\mathcal{F}_{\mathrm{B}}$ is isomorphic to the tensor product $\otimes_{\sigma, \mathbf{p} \in \Lambda_{\ell}^{*}} \mathcal{F}_{\mathbf{p}, \sigma}^{\mathrm{B}}$ where $\mathcal{F}_{\mathbf{p}, \sigma}^{\mathrm{B}}$ is the boson Fock space constructed on the one-dimensional Hilbert space $\mathcal{H}_{\mathbf{p}, \sigma}=\left\{c e^{i \mathbf{p} \cdot \mathbf{x}} \otimes e_{\sigma}\right\}_{c \in \mathbb{C}}$, where $e_{1}=(1,0,0)$, $e_{2}=(0,1,0), e_{3}=(0,0,1)$.

Let

$$
p_{\ell}(\beta, \mu)=\frac{1}{\beta V_{\ell}} \ln \operatorname{Tr}_{\mathcal{F}_{\mathrm{B}}} \exp \left(-\beta\left(\hat{H}_{\ell}-\mu \hat{N}\right)\right)
$$

be the grand-canonical pressure at finite volume, corresponding to $\hat{H}_{\ell}$. Following standard arguments [5], it is not hard to prove that for all $\mu \in \mathbb{R}$

$$
\lim _{V_{\ell} \rightarrow \infty} p_{\ell}(\beta, \mu)=p(\beta, \mu)<\infty
$$

In other words the system is a superstable one. Thus the domain of definition of the limit pressure $p(\beta, \mu)$ is

$$
\mathcal{D}(p)=\left\{(\beta, \mu) \in \mathbb{R}^{2}: \beta>0, \mu \in \mathbb{R}\right\} .
$$

If $\hat{H}_{\ell}(\mu)=\hat{H}_{\ell}-\mu \hat{N}$, the equilibrium Gibbs state ( grand canonical ensemble) $\langle-\rangle_{\hat{H}_{\ell}(\mu)}$ is defined as

$$
\langle\hat{A}\rangle_{\hat{H}_{\ell}(\mu)}=\frac{\operatorname{Tr}_{\mathcal{F}_{\mathrm{B}}} \hat{A} \exp \left(-\beta \hat{H}_{\ell}(\mu)\right)}{\operatorname{Tr}_{\mathcal{F}_{\mathrm{B}}} \exp \left(-\beta \hat{H}_{\ell}(\mu)\right)}
$$

for any operator $\hat{A}$ acting on the symmetric Fock space. Finally, the total density of particles $\rho(\mu)$ for infinite volume is defined as

$$
\lim _{V_{\ell} \rightarrow \infty}\left\langle\frac{\hat{N}}{V_{\ell}}\right\rangle_{\hat{H}_{\ell}(\mu)}=\lim _{V_{\ell} \rightarrow \infty} \rho_{\ell}(\mu)=\rho(\mu),
$$

and the density of particles $\rho_{\mathbf{0}, \sigma}(\beta, \mu)$ associated to the mode $(\mathbf{0}, \sigma)$ is defined as

$$
\lim _{V_{\ell} \rightarrow \infty}\left\langle\frac{\hat{n}_{\mathbf{0}, \sigma}}{V_{\ell}}\right\rangle_{\hat{H}_{\ell}(\mu)}=\lim _{V_{\ell} \rightarrow \infty} \rho_{\mathbf{0}, \sigma, \ell}(\beta, \mu)=\rho_{\mathbf{0}, \sigma}(\beta, \mu) .
$$

We shall say that the system undergoes a macroscopic occupation of the single particle mode $(\mathbf{0}, \sigma)$ (BEC) if $\rho_{\mathbf{0}, \sigma}(\beta, \mu)>0$. Independent on temperature BEC is named non-conventional BEC.

\subsection{Case $\gamma=\gamma_{1,2}=\gamma_{1,3}=\gamma_{2,3}$}

Let $s$ and $t$ be defined as

$$
s:=-3 \gamma^{2} / 4, \quad t:=\gamma^{3} / 4
$$

Proposition 2.1. For $a>-3 t /(2 s)>0$ the quadratic form given as

$$
\begin{array}{r}
q(x, y, z)=-\left(\lambda_{1}+\mu\right) x_{1}+a x_{1}^{2}-\left(\lambda_{3}+\mu\right) x_{3}+a x_{3}^{2} \\
-\mu x_{2}+a x_{2}^{2}+\gamma\left(x_{1} x_{2}+x_{2} x_{3}+x_{1} x_{3}\right), \\
x_{1}, x_{2}, x_{3} \in \mathbb{R}
\end{array}
$$

is a convex function.

Proof. Eq. (3) can be written in the following form $q(\mathbf{x})=\frac{1}{2} \mathbf{x} Q \mathbf{x}^{T}+\mathbf{d} \mathbf{x}^{T}, \quad \mathbf{d}, \quad \mathbf{x} \in \mathbb{R}^{3}$ where $Q$ is the corresponding Hessian matrix. Therefore, $q$ is a convex function if $Q$ is a positive definite matrix, i.e. $\triangle(a):=\operatorname{det}(Q)>0$. It yields to the inequality

$$
\frac{1}{8} \triangle(a)=a^{3}-\frac{3 \gamma^{2} a}{4}+\frac{\gamma^{3}}{4}=a^{3}+a s+t>0 .
$$

We are interested in finding the values of $a$ for which ineq. (4) holds. In this sense, since the cubic equation

$$
a^{3}+a s+t=0
$$

has a double real root and a simple real root given by $a_{1,2}=-3 t /(2 s)>0, \quad a_{3}=-4 s^{2} /(9 t)<0$, respectively, we have that for $a>-3 t /(2 s)=\gamma / 2$ inequality (4) holds.

Therefore, under conditions of the last proposition, the quadratic form $q(\mathbf{x})$ is a convex function attaining a global minimum at $\mathbf{x}^{*}$ satisfying the equations system $\nabla q(\mathbf{x})=\mathbf{0}$. In this case the Hessian matrix $\mathcal{H}(\mathbf{x})=Q / 2$.

Proposition 2.2. Under the conditions of Proposition 2.1 , the quadratic form $q(\mathbf{x})$ attains its global minimum at $\left(x_{1}^{*}, x_{2}^{*}, x_{3}^{*}\right)$ given by

$$
\begin{aligned}
\triangle(a) x_{1}^{*} & =\left(4 a^{2}-4 \gamma a+\gamma^{2}\right) \mu-4 \lambda_{1} a^{2} \\
& -2 a \lambda_{3} \gamma+\gamma^{2}\left(\lambda_{3}-\lambda_{1}\right)=P_{1}(a) \\
\triangle(a) x_{2}^{*}= & \left(4 a^{2}-4 \gamma a+\gamma^{2}\right) \mu-2 a \gamma\left(\lambda_{1}+\lambda_{3}\right) \\
+ & \gamma^{2}\left(\lambda_{1}+\lambda_{3}\right)=P_{2}(a) \\
\triangle(a) x_{3}^{*} & =\left(4 a^{2}-4 \gamma a+\gamma^{2}\right) \mu-4 \lambda_{3} a^{2} \\
& -2 a \lambda_{1} \gamma+\gamma^{2}\left(\lambda_{1}-\lambda_{3}\right)=P_{3}(a) .
\end{aligned}
$$

In this sense, being $\mathcal{A}_{\sigma}=\left\{a \in \mathbb{R}^{+}: P_{\sigma}(a)>0\right\}$, with $\sigma=1,2,3$, and $\mathcal{B}=\left\{a \in \mathbb{R}^{+}: \triangle(a)>0\right\}$, it is not hard to see that

Proposition 2.3. For all $x_{\sigma}^{*}, \sigma=1,2,3$ in Proposition 2.2 and $a \in \mathcal{A}_{\sigma} \cap \mathcal{B}$, we have $x_{\sigma}^{*}>0$. 


\subsection{Approximating Hamiltonian}

To prove the existence of a BEC in the case of the many particle system whose energy operator is given by eq. (1), the Fock space $\mathcal{F}_{\mathrm{B}}$ is decomposed as the tensorial product $\mathcal{F}_{\mathbf{0}} \otimes \mathcal{F}_{\mathrm{B}}^{\prime}$, where $\mathcal{F}_{\mathbf{0}}$ is the symmetric tensorial algebra constructed in the space of constants (mode $\mathbf{0}$ ) and $\mathcal{F}_{B}^{\prime}$ is its complementary orthogonal tensorial algebra of functions in $L^{2}\left(\Lambda_{\ell}\right)$. For any complex number $\eta \in \mathbb{C}$ let us consider a coherent vector in $\mathcal{F}_{\mathbf{0}}$,

$$
\phi_{\eta}=e^{-V_{\ell}|\eta|^{2} / 2} \sum_{n=0}^{\infty} \frac{1}{n !}\left(V_{\ell}^{\frac{1}{2}} \eta\right)^{n}\left(a_{0}^{\dagger}\right)^{n} \phi_{0}
$$

where $\phi_{0} \in \mathcal{F}_{0}$. Thus, for any operator $\hat{A}$ in $\mathcal{F}_{\mathrm{B}}$, it is possible to construct an operator $\hat{A}(\gamma)$ such that

$$
\left\langle\Psi_{1}^{\prime}, \hat{A}(\eta) \Psi_{2}^{\prime}\right\rangle_{\mathcal{F}_{\mathrm{B}}^{\prime}}=\left\langle\Psi_{1}^{\prime} \otimes \phi_{\eta}, \hat{A} \Psi_{2}^{\prime} \otimes \phi_{\eta}\right\rangle_{\mathcal{F}_{\mathrm{B}}},
$$

where $\Psi_{1}^{\prime}, \Psi_{2}^{\prime} \in \mathcal{F}_{\Lambda}^{\prime}$. The transition from the operator $\hat{A}$ to the operator $\hat{A}(\gamma)$ is called approximation of Bogolyubov and it consists in replacing the operators $\hat{a}_{0}$ and $\hat{a}_{0}^{\dagger}$ by complex numbers $\eta \sqrt{V_{\ell}}$ and $\bar{\eta} \sqrt{V_{\ell}}$, respectively, in the operator $\hat{A}$ written in the normal form.

$$
\begin{aligned}
& \hat{H}_{\ell}^{\mathrm{app}}\left(\eta_{1}, \eta_{2}, \eta_{3}, \mu\right)=\hat{H}_{\ell}^{0 \prime}(\mu)+\frac{a}{V_{l}} \sum_{\sigma, \mathbf{p} \neq \mathbf{0}}\left(\hat{a}_{\sigma, \mathbf{p}}^{\dagger}\right)^{2} \hat{a}_{\sigma, \mathbf{p}}^{2} \\
& +V_{\ell} g\left(\frac{\hat{N}^{\prime}}{V_{\ell}}\right)+V_{\ell} \sum_{\sigma}\left(\lambda_{\ell}(\mathbf{0}, \sigma)-\mu\right)\left|\eta_{\sigma}\right|^{2} \\
& +V_{\ell} a \sum_{\sigma}\left|\eta_{\sigma_{1}}\right|^{4}+V_{\ell} \sum_{\sigma_{1}<\sigma_{2}} \gamma_{\sigma_{1}, \sigma_{2}}\left|\eta_{\sigma_{1}}\right|^{2}\left|\eta_{\sigma_{2}}\right|^{2} .
\end{aligned}
$$

$$
\text { Here } \hat{H}_{\ell}^{0 \prime}(\mu)=\sum_{\sigma, \mathbf{p} \in \Lambda_{\ell}^{*} \backslash\{\mathbf{0}\}}\left(\lambda_{\ell}(\mathbf{p}, \sigma)-\mu\right) \hat{n}_{\mathbf{p}, \sigma} .
$$

Theorem 2.4.

$$
\operatorname{Tr}_{\mathcal{F}_{\mathbf{0}}^{\prime}} e^{-\beta \hat{H}_{\ell}^{\mathrm{appr}}\left(\eta_{1}, \eta_{2}, \eta_{3}, \mu\right)} \leq \operatorname{Tr}_{\mathcal{F}_{\mathrm{B}}} e^{-\beta \hat{H}_{\ell}(\mu)} .
$$

Proof. The proof follows from the definition of trace and the definition of Bogolyubov approximation.

Theorem 2.5. The systems with Hamiltonians $\hat{H}_{\ell}(\mu)$ and $\hat{H}_{\ell}^{\text {appr }}\left(\eta_{1}, \eta_{2}, \eta_{3}, \mu\right)$ are thermodynamically equivalent in the Wentzel sense, i.e.

$$
\lim _{V_{\ell} \rightarrow \infty} \sup _{\left|\eta_{\sigma}\right|: \eta_{\sigma} \in \mathbb{C}, \sigma=1,2,3} p_{l}^{\text {appr }}\left(\beta, \eta_{1}, \eta_{2}, \eta_{3}, \mu\right)=p(\beta, \mu),
$$

where

$$
\begin{aligned}
& p_{\ell}^{\text {appr }}\left(\beta, \eta_{1}, \eta_{2}, \eta_{3}, \mu\right) \\
\equiv & \frac{1}{\beta V_{\ell}} \ln \operatorname{Tr}_{\otimes_{p \in \Lambda^{*} \backslash\{0\}} \mathcal{F}_{\mathbf{p}}^{\text {B }}} \exp \left[-\beta\left(\hat{H}_{\ell}^{\text {appr }}\left(\eta_{1}, \eta_{2}, \eta_{3}, \mu\right)\right)\right] .
\end{aligned}
$$

Proof. Applying a well-known Bogolyubov inequality for pressures and the last theorem, obtain

$$
\begin{aligned}
0 & \leq p_{\ell}(\beta, \mu)-p_{\ell}^{\mathrm{appr}}\left(\eta_{1}, \eta_{2}, \eta_{3}, \mu\right) \\
& \left.\leq \frac{1}{V_{l}}\left\langle\hat{H}_{\ell}^{\mathrm{appr}}\left(\eta_{1}, \eta_{2}, \eta_{3}, \mu\right)\right)-\hat{H}_{\ell}(\mu)\right\rangle_{\hat{H}_{l}(\mu)} \\
& \left.=\delta_{\ell}\left(\eta_{1}, \eta_{2}, \eta_{3}, \mu\right)\right) .
\end{aligned}
$$

The difference $\delta \equiv \hat{H}_{\ell}^{\text {appr }}\left(\eta_{1}, \eta_{2}, \eta_{3}, \mu\right)-\hat{H}_{\ell}(\mu)$ can be written in the following form:

$$
\begin{aligned}
\delta & =-V_{\ell}\left(\lambda_{1}+\mu\right)\left|\eta_{1}\right|^{2}+a V_{\ell}\left|\eta_{1}\right|^{4} \\
& -V_{\ell}\left(\lambda_{3}+\mu\right)\left|\eta_{3}\right|^{2}+a V_{\ell}\left|\eta_{3}\right|^{4}-V_{\ell} \mu\left|\eta_{2}\right|^{2} \\
& +V_{\ell} a\left|\eta_{2}\right|^{4}+V_{\ell} h_{\ell}\left(\hat{\rho}_{\mathbf{0}, 1}, \hat{\rho}_{\mathbf{0}, 2}, \hat{\rho}_{\mathbf{0}, 3}\right),
\end{aligned}
$$

where

$$
\begin{gathered}
h_{\ell}\left(x_{1}, x_{2}, x_{3}\right)=\sum_{i=1}^{3}\left(\lambda_{\ell}(\mathbf{0}, \sigma)+\mu\right) x_{\sigma} \\
-a \sum_{i=1}^{3} x_{\sigma}\left(x_{\sigma}-\frac{1}{V_{\ell}}\right)-\sum_{\sigma_{1}<\sigma_{2}} \gamma_{\sigma_{1}, \sigma_{2}} x_{\sigma_{1}} x_{\sigma_{2}}
\end{gathered}
$$

is a concave function under assumptions of the Proposition 2.1, and $\hat{\rho}_{\mathbf{0}, \sigma}=\hat{n}_{\mathbf{0}, \sigma} / V_{\ell}, \sigma=1,2,3$. Therefore,

$$
\begin{aligned}
0 & \leq \delta_{\ell}\left(\eta_{1}, \eta_{2}, \eta_{3}, \mu\right) \\
& =-\left(\lambda_{1}+\mu\right)\left|\eta_{1}\right|^{2}+a\left|\eta_{1}\right|^{4}-\left(\lambda_{3}+\mu\right)\left|\eta_{3}\right|^{2}+a\left|\eta_{3}\right|^{4} \\
& -\mu\left|\eta_{2}\right|^{2}+a\left|\eta_{2}\right|^{4}+\left\langle h_{\ell}\left(\hat{\rho}_{\mathbf{0}, 1}, \hat{\rho}_{\mathbf{0}, 2}, \hat{\rho}_{\mathbf{0}, 3}\right)\right\rangle_{\hat{H}_{\ell}(\mu)} \\
& =q\left(\left|\eta_{1}\right|,\left|\eta_{2}\right|,\left|\eta_{3}\right|\right)+\left\langle h_{\ell}\left(\hat{\rho}_{\mathbf{0}, 1}, \hat{\rho}_{\mathbf{0}, 2}, \hat{\rho}_{\mathbf{0}, 3}\right)\right\rangle_{\hat{H}_{\ell}(\mu)}
\end{aligned}
$$

for $q(x, y, z)$ defined in eq. (2). Using standard arguments of spectral theory of unbounded operators [6], it is not hard to see that

$$
\left\langle h_{\ell}\left(\hat{\rho}_{\mathbf{0}, 1}, \hat{\rho}_{\mathbf{0}, 2}, \hat{\rho}_{\mathbf{0}, 3}\right)\right\rangle_{\hat{H}_{\ell}(\mu)} \leq \sup _{\left\{x_{\sigma} \in[0, \infty)\right\}} h_{\ell}\left(x_{1}, x_{2}, x_{3}\right) .
$$

Then

$$
\begin{aligned}
0 & \leq \lim _{V_{\ell} \rightarrow \infty\left\{\eta_{\sigma} \in \mathbb{C}\right\}} \delta_{\ell}\left(\eta_{1}, \eta_{2}, \eta_{3}, \mu\right) \\
& \leq \lim _{V_{\ell} \rightarrow \infty\left\{\eta_{\sigma} \in \mathbb{C}\right\}} q\left(\left|\eta_{1}\right|,\left|\eta_{2}\right|,\left|\eta_{3}\right|\right. \\
& \left.+\sup _{\left\{x_{\sigma} \in[0, \infty)\right\}} h_{\ell}\left(x_{1}, x_{2}, x_{3}\right)\right),
\end{aligned}
$$

where the infimum is taken over $\left(\left|\eta_{1}\right|,\left|\eta_{2}\right|,\left|\eta_{3}\right|\right)$ at all sides of these inequalities. Straightforward calculations yield to

$$
\begin{aligned}
& \lim _{V_{\ell} \rightarrow \infty} \inf _{\left\{\eta_{\sigma} \in \mathbb{C}\right\}} q\left(\left|\eta_{1}\right|,\left|\eta_{2}\right|,\left|\eta_{3}\right|\right) \\
=- & \lim _{V_{\ell} \rightarrow \infty} \sup _{\left\{x_{\sigma} \in[0, \infty)\right\}} h_{\ell}\left(x_{1}, x_{2}, x_{3}\right) .
\end{aligned}
$$

Thus

$$
\begin{aligned}
0 & \leq \lim _{V_{\ell} \rightarrow \infty} \inf _{\left\{\eta_{\sigma} \in \mathbb{C}\right\}} \delta_{\ell}\left(\eta_{1}, \eta_{2}, \eta_{3}, \mu\right) \\
& \leq \lim _{V_{\ell} \rightarrow \infty} \inf _{\left\{\eta_{\sigma} \in \mathbb{C}\right\}}\left\{-\left(\lambda_{1}+\mu\right)\left|\eta_{1}\right|^{2}+a\left|\eta_{1}\right|^{4}\right. \\
& \left.-\left(\lambda_{3}+\mu\right)\left|\eta_{3}\right|^{2}+a\left|\eta_{3}\right|^{4}-\mu\left|\eta_{2}\right|^{2}+a\left|\eta_{2}\right|^{4}\right\} \\
& +\lim _{V_{\ell} \rightarrow \infty} \sup _{\left\{x_{\sigma} \in[0, \infty)\right\}} h_{\ell}\left(x_{1}, x_{2}, x_{3}\right)=0 .
\end{aligned}
$$

Then the proof of the theorem follows.

Theorem 2.6. Let $\hat{H}_{\ell}^{\mathrm{MF}^{\prime}}$ be the operator defined as

$$
\hat{H}_{\ell}^{\mathrm{MF}}=\sum_{\mathbf{p} \in \Lambda_{\ell}^{*} \backslash\{\mathbf{0}\}, \sigma} \lambda_{\ell}(\mathbf{0}, \sigma) \hat{n}_{\mathbf{0}, \sigma}+V_{\ell} g\left(\frac{\hat{N}^{\prime}}{V_{\ell}}\right) .
$$

Then, for $(\mu, \beta) \in \mathcal{D}(p)$ we have

$$
p(\beta, \mu)=\inf _{x_{1}, x_{2}, x_{3} \in[0, \infty]} q\left(x_{1}, x_{2}, x_{3}\right)+p^{\mathrm{MF}^{\prime}}(\beta, \mu),
$$


where the limit grand canonical pressure

$$
p^{\mathrm{MF}^{\prime}}(\beta, \mu)=\sup _{r \geq 0} \inf _{\alpha \leq 0}\left\{(\mu-\alpha) r-g(r)+p^{\mathrm{p}^{\prime}}(\beta, \alpha)\right\}
$$

and $p^{\mathrm{p}^{\prime}}(\beta, \alpha)$ is the limit grand canonical pressure for the ideal Bose gas with exclusion of the zero mode at chemical potential $\alpha \leq 0$.

Proof. This follows from the standard arguments already used in the works $[5,6,7]$.

We want to find a set $\left\{x_{\sigma}^{*}>0, \sigma=1,2,3\right\}$ such that

$$
q\left(x_{1}^{*}, x_{2}^{*}, x_{3}^{*}\right)=\inf _{x_{1}, x_{2}, x_{3} \in[0, \infty]} q\left(x_{1}, x_{2}, x_{3}\right) .
$$

\subsection{Limit pressure and non-conventional BEC}

Considering that for the Hamiltonian given by eq. (1) $a$ is positive and $-3 t /(2 s)=\gamma / 2>0$ we get

Theorem 2.7. Assume that $\lambda_{3} \in\left(0, \lambda_{1}\right), \mu>\left(\lambda_{1}+\right.$ $\left.\lambda_{3}\right) /(2 a-\gamma)$ and $a>\max \left\{\gamma / 2, c_{+}\right\}$, where

$$
c_{+}=\frac{\gamma}{2\left(\mu+\lambda_{3}\right)}\left(\mu+\frac{\lambda_{1}}{2}+\left|\frac{\lambda_{1}}{2}-\lambda_{3}\right|\right) .
$$

Then all $x_{\sigma}^{*}$ defined in Proposition 2.2 are strictly positive.

Proof. We have that $\triangle(a)=8\left(a-\frac{\gamma}{2}\right)^{2}(a+\gamma)>0$ with $a>0, a \neq \gamma / 2$. On the other hand, note that

$$
\chi(u, v)=\frac{u^{2}}{4}-u v+v^{2}=\left(\frac{u}{2}-v\right)^{2} \geq 0 .
$$

Our purpose is to determine the range of values of $a$ for which $P_{1}(a)>0, P_{2}(a)>0, P_{3}(a)>0$ in Proposition 2.2. The numerator $P_{1}(a)$ in eq. (6) can be rewritten as $P_{1}(a)=4\left(\mu+\lambda_{1}\right) a^{2}-2 \gamma\left(2 \mu+\lambda_{3}\right) a+\gamma^{2} \mu+\gamma^{2}\left(\lambda_{3}-\lambda_{1}\right)$.

Function $P_{1}(a)$ in eq. (6) satisfies the inequality

$\frac{P_{1}(a)}{4}=\left(\mu+\lambda_{1}\right) a^{2}-\frac{\gamma}{2}\left(2 \mu+\lambda_{3}\right) a+\frac{\gamma^{2}}{4}\left(\mu+\lambda_{3}-\lambda_{1}\right)>0$ for $a>a_{+}$, where

$$
\begin{aligned}
a_{ \pm} & =\frac{\gamma}{2\left(\mu+\lambda_{1}\right)}\left(\mu+\frac{\lambda_{3}}{2} \pm \sqrt{\chi\left(\lambda_{3}, \lambda_{1}\right)}\right) \\
& =\frac{\gamma}{2\left(\mu+\lambda_{1}\right)}\left(\mu+\frac{\lambda_{3}}{2} \pm\left|\frac{\lambda_{3}}{2}-\lambda_{1}\right|\right),
\end{aligned}
$$

i.e.

$$
\begin{gathered}
a_{+}=\frac{\gamma}{2\left(\mu+\lambda_{1}\right)}\left(\mu+\lambda_{1}\right)=\frac{\gamma}{2}>0, \\
a_{-}=\frac{\gamma}{2\left(\mu+\lambda_{1}\right)}\left(\mu+\lambda_{3}-\lambda_{1}\right)
\end{gathered}
$$

are the real roots of $P_{1}(a)=0$. In this case

$$
x_{1}^{*}=[\triangle(a)]^{-1} P_{1}(a)>0
$$

when $a>\gamma / 2$. On the other hand, for $P_{2}(a)$ in eq. (7) and $\mu>\left(\lambda_{1}+\lambda_{3}\right) /(2 a-\gamma)$, we get that

$P_{2}(a)=4 \mu a^{2}-2 \gamma\left(2 \mu+\lambda_{1}+\lambda_{3}\right) a+\gamma^{2}\left(\mu+\lambda_{1}+\lambda_{3}\right)>0$ for $a>\max \left\{b_{+}, b_{-}\right\}$, where

$$
b_{+}=\frac{\gamma\left(\mu+\lambda_{1}+\lambda_{3}\right)}{2 \mu}>0, \quad b_{-}=\frac{\gamma}{2}>0
$$

are the real roots of $P_{2}(a)=0$. Thus,

$$
x_{2}^{*}=[\triangle(a)]^{-1} P_{2}(a)>0
$$

for $a>\gamma / 2$. Finally, for $P_{3}(a)$ in eq. (8) we obtain that $\frac{P_{3}(a)}{4}=\left(\mu+\lambda_{3}\right) a^{2}-\frac{\gamma}{2}\left(2 \mu+\lambda_{1}\right) a+\frac{\gamma^{2}}{4}\left(\mu+\lambda_{1}-\lambda_{3}\right)>0$ for $a>c_{+}$, where

$$
\begin{aligned}
c_{ \pm} & =\frac{\gamma}{2\left(\mu+\lambda_{3}\right)}\left(\mu+\frac{\lambda_{1}}{2} \pm \sqrt{\chi\left(\lambda_{1}, \lambda_{3}\right)}\right) \\
& =\frac{\gamma}{2\left(\mu+\lambda_{3}\right)}\left(\mu+\frac{\lambda_{1}}{2} \pm\left|\frac{\lambda_{1}}{2}-\lambda_{3}\right|\right)
\end{aligned}
$$

are the real roots of $P_{3}(a)=0$. In this case we obtain that,

$$
x_{3}^{*}=[\triangle(a)]^{-1} P_{3}(a)>0
$$

for $a>\max \left\{\gamma / 2, c_{+}\right\}$. Therefore $x_{\sigma}^{*}>0, \quad \sigma=1,2,3$ for $a>\max \left\{\gamma / 2, c_{+}\right\}, \mu>\left(\lambda_{1}+\lambda_{3}\right) /(2 a-\gamma)$ and $c_{+} \in(0, \infty)$.

Corollary 2.8. Under conditions of the Theorem (2.7) it follows that:

$$
p(\beta, \mu)=q\left(x_{1}^{*}, x_{2}^{*}, x_{3}^{*}\right)+p^{\mathrm{MF}^{\prime}}(\beta, \mu),
$$

where

$$
\left\{\begin{array}{l}
x_{1}^{*}=\frac{\left(\mu+\lambda_{1}\right)\left(a-a_{-}\right)}{2(2 a-\gamma)(a+\gamma)} \\
x_{2}^{*}=\frac{\mu\left(a-b_{+}\right)}{(2 a-\gamma)(a+\gamma)} \\
x_{3}^{*}=\frac{\left(\mu+\lambda_{3}\right)\left(a-c_{+}\right)\left(a-c_{-}\right)}{(2 a-\gamma)(a+\gamma)} .
\end{array}\right.
$$

Corollary 2.9. Under conditions of the Theorem (2.7) the system given by Hamiltonian (1) displays simultaneous BEC and the amount of condensate corresponding to each internal level of the ground state is given by

$$
\rho_{\mathbf{0}, 1}=x_{1}^{*}, \rho_{\mathbf{0}, 2}=0, \rho_{\mathbf{0}, 3}=x_{3}^{*} .
$$

\subsection{Vanishing spectrum gaps}

Taking the limits $\lambda_{1} \downarrow 0, \lambda_{3} \downarrow 0$ we get $a_{+}=a_{-}=$ $b_{+}=b_{-}=c_{+}=c_{-}=\gamma / 2$, then

$$
\rho_{\mathbf{0}, 1}=\frac{\mu}{4(a+\gamma)}, \rho_{\mathbf{0}, 2}=0, \rho_{\mathbf{0}, 3}=\frac{\mu\left(a-\frac{\gamma}{2}\right)}{2(a+\gamma)} .
$$

In this case the limit grand canonical pressure is given by the expression

$$
p(\beta, \mu)=\left(\frac{\mu}{2(a+\gamma)}\right)^{2} f(a, \gamma)+p^{\mathrm{MF}^{\prime}}(\beta, \mu),
$$


where

$$
\begin{aligned}
f(a, \gamma) & =\gamma\left(\frac{1}{2}+\frac{3}{2}(a-\gamma)\right)-\left(\frac{7}{4} a+3 \gamma\right) \\
& -\left(a-\frac{\gamma}{2}\right)\left(a^{2}-a\left(\frac{\gamma}{2}+2\right)-2 \gamma\right)
\end{aligned}
$$

It is not hard to see, that for $\mu=0$ we have $p(\beta, 0)=$ $p^{\mathrm{MF}^{\prime}}(\beta, 0)$ and a macroscopic occupation of the ground state does not occur. As an example, taking $g(x)=x^{2}$ we obtain

$$
\begin{aligned}
p(\beta, \mu) & =\left(\frac{\mu}{2(a+\gamma)}\right)^{2} f(a, \gamma) \\
& +\sup _{\alpha \leq 0}\left\{p^{\mathrm{p}^{\prime}}(\beta, \alpha)+\frac{(\mu-\alpha)^{2}}{2 a}\right\},
\end{aligned}
$$

where $p^{\mathrm{p}^{\prime}}(\beta, \alpha)$ is the pressure of the ideal Bose gas with exclusion of a zero mode.

\section{Conclusions}

In section 2 we studied the system of atoms whose quantum states have three internal degrees of freedom. In this case a couple of energies $\left\{-\lambda_{1},-\lambda_{3}\right\}$, with $\lambda_{1}>$ $\lambda_{3}>0$, associated with two of the ground states internal levels, generalize our previous results on the spin-0 and spin- $1 / 2$ Bose systems. We derive an exact expression for the pressure by applying approximating Hamiltonians and large deviations approaches. Also we prove the emergence of non-conventional BEC ( independent on temperature) for a range of values of the intrastate collisions parameter $a$ with $a>\max \left\{\gamma / 2, c_{+}\right\}$, where $\gamma$ is the coupling constant for interstate collisions, $c_{+}$is given by eq. (14) and the chemical potential $\mu$ satisfies the inequality $\mu>\left(\lambda_{1}+\lambda_{3}\right) /(2 a-\gamma)$.

\section{Acknowledgements}

This work was partially supported by project ACT1112 (Red de Análisis Estocástico y Aplicaciones: sistemas abiertos, energía y dinámica de la información) financed by Conicyt, Chile.

\section{REFERENCES}

[1] J. Ieda, M. Wadati. Exact Nonlinear Dynamics in Spinor Bose-Einstein Condensates, in Nonlinear Dynamics, Edited by Todd Evans, 2010. Publisher: InTech.

[2] Ming-Shien Chang. Coherent Spin Dynamics of a Spin-1 Bose-Einstein, Ph.D Thesis, School of Physics Georgia Institute of Technology, 2006.

[3] T.L. Ho. Spinor Bose condensates in optical traps, Phys. Rev. Lett. 81, 742 (1998).

[4] T. Ohmi, K. Machida. Bose-Einstein condensation with internal degrees of freedom in alkali atom gases, J. Phys. Soc. Jpn. 67, 1822 (1998).
[5] M. Corgini, C. Rojas-Molina, D.P. Sankovich. Coexistence of non-conventional condensates in two-level Bose atom systems. International Journal of Modern Physics B, Vol. 22, No. 27: 4799-4815, 2008.

[6] M. Corgini, D.P. Sankovich. Bogolyubov approximation for a diagonal model of an interacting Bose-gas. Physics Letters A , 360 (3) : 419-422, 2007.

[7] M. Corgini, D.P. Sankovich. Soluble model of Boseatoms with two level internal structure: nonconventional Bose-Einstein condensation. Condensed Matter Physics, Vol. 13, No.4, 43003,1-11, 2010.

[8] D.W. Robinson. Bose-Einstein condensation with attractive boundary conditions, Commun. Math. Phys. 50, 53-59, 1976.

[9] L.J. Landau, I.F. Wilde. On the Bose-Einstein condensation of an ideal gas, Commun. Math. Phys. 70, 43-51, 1979.

[10] L. Vandevenne, A. Verbeure, V.A. Zagrebnov. Equilibrium states for the Bose-gas, J. Math. Phys. 45, no. 4, 1606-1622, 2004.

[11] F. Dalfovo, S. Giorgini, L.P. Pitaevskii, S. Stringari. Theory of Bose-Einstein condensation in trapped gases, Rev. Mod. Phys. 71, 463-512, 1999.

[12] J.O. Indekeu, J.M.J. van Leeuwen. Wetting, prewetting and surface transitions in type-I superconductors, Physica C, 251, 290-306, 1995.

[13] F. London. Superfluids, vol. II, Wiley, New York, 1954.

[14] J. Lauwers, A. Verbeure, V.A. Zagrebnov. Proof of Bose-Einstein condensation for interacting gases with a one-particle spectral gap, J. Phys. A: Math. Gen. 36, 169-174, 2002.

[15] J. Lauwers, A. Verbeure, V.A. Zagrebnov. BoseEinstein condensation for homogeneous interacting systems with a one-particle spectral gap, J. Stat. Phys. 112, 397-420, 2002.

[16] J. Lauwers. Quantum uctuations and Bose-Einstein condensation in systems with a one-particle spectral gap, Ph.D. thesis, Katholieke Universiteit Leuven, 2003.

[17] T.C. Dorlas, J.T. Lewis, J.V. Pulé. The full diagonal model of a Bose gas, Commun. Math. Phys. 156, 37 65, 1993.

[18] J-B. Bru, V.A. Zagrebnov. Exactly soluble model with two kinds of Bose-Einstein condensations, Physica A 268, 309-325, 1999.

[19] J-B. Bru, V.A. Zagrebnov. A model with coexistence of two kinds of Bose condensations, J. Phys. A: Math. Gen. 33 (2000), 449-464.

[20] V.A. Zagrebnov, J-B. Bru. The Bogoliubov model of weakly imperfect Bose gas, Phys. Rep. 350, no. 56, 291434, 2001.

[21] E.B. Davies. The Thermodynamic Limit for an Imperfect Boson Gas, Commun. Math. Phys. 28, 69-86, 1972. 